\title{
Expression of extracellular matrix metalloproteinase inducer and fascin in urinary bladder cancer: Correlation with clinicopathological characteristics
}

\author{
DALIA MOHAMED ABD EL-REHIM ${ }^{1}$, NEHAD MOHAMED REDA ABD EL-MAQSOUD ${ }^{1}$, \\ AMR MOHAMED ABD EL-HAMID ${ }^{2}$, TAREK KHALAF FATH EL-BAB ${ }^{2}$ and EHAB MOHAMED GALAL ${ }^{2}$ \\ Departments of ${ }^{1}$ Pathology and ${ }^{2}$ Urology, Faculty of Medicine, Minia University, Minia 61111, Egypt
}

Received September 6, 2012; Accepted December 19, 2012

DOI: $10.3892 / \mathrm{mco} .2013 .57$

\begin{abstract}
Expression of extracellular matrix metalloproteinase inducer (EMMPRIN) and fascin have recently received considerable attention as emerging key markers of cell invasion and metastasis in various types of cancers. The present study aimed to characterize the expression of EMMPRIN and fascin in a cohort of bladder cancer patients and to verify the association with clinicopathological characteristics. Immunohistochemical analysis of EMMPRIN and fascin was performed in 125 bladder cancer specimens, including 86 transitional cell carcinoma (TCC) and 39 squamous cell carcinoma (SCC) cases. Overexpression of EMMPRIN and fascin was detected in 71.2 and $83.2 \%$ of bladder carcinomas, respectively, while neither was detectable in the normal adjacent epithelium. In SCC, EMMPRIN and fascin expression scores were found to be higher compared to TCC cases $(\mathrm{P}=0.031$ and 0.053 , respectively). Higher EMMPRIN and fascin immunoscores markedly correlated with tumor pathological ( $\mathrm{pT}$ ) stage in TCC $(\mathrm{P}<0.001)$ and $\mathrm{SCC}(\mathrm{P}=0.004$ and 0.001 , respectively). High EMMPRIN expression was strongly associated with advanced grades in TCC and SCC ( $\mathrm{P}=0.003$ and 0.002 , respectively). Cases were then stratified, according to the joint expression status of EMMPRIN and fascin, into four immunoprofiles. A highly significant association was found between various tumor immunoprofiles and pathological stage. Of those, the EMMPRIN-/fascin-positive immunophenotype was closely associated with pT3 and pT4 tumors. The findings suggested an association between increased EMMPRIN and fascin expression and increased progression of bladder cancer. Additionally,
\end{abstract}

Correspondence to: Dr Dalia Mohamed Abd El-Rehim, Department of Pathology, Faculty of Medicine, Minia University, Minia 61111, Egypt

E-mail: dalia_abdelrehim@hotmail.com

Key words: expression of extracellular matrix metalloproteinase inducer, fascin, immunohistochemistry, bladder carcinoma, Egyptian patients these two markers may act in concert to mediate a more aggressive behavior through enhanced tumor cell invasion.

\section{Introduction}

Bladder cancer is a global health problem. Its incidence ranks ninth worldwide (1). In Egypt, the situation is critical. Bladder cancer has been and remains one of the most prevalent malignancies, accounting for $12.22 \%$ of the incident cancers and representing the main bulk of the urinary system malignancy (2). An aggressive form of this type of cancer, schistosomiasis-associated bladder cancer, has been encountered among bladder cancer patients. During the past decade, certain changes have been observed in the features of bladder cancer associated with schistosomiasis (bilharziasis) in Egypt, with a decrease in the frequency of squamous cell carcinoma (SCC) and an increase in the incidence of transitional cell carcinoma (TCC) cases (3).

Histopathological evaluation of tissues is the basis for the grading and pathological staging of urinary bladder cancer, delineating treatment strategy and predicting subsequent clinical outcome. However, reliable prognostic information regarding the biological behavior of these tumors is limited (4).

In addition, bladder cancer has markedly different behavioral characteristics, however, patients with the same disease stage may have divergent clinical course and different outcomes following the same treatment regimen (5).

Therefore, new molecular markers that provide additional objective information on the biological behavior of these tumors may allow a more precise assessment and achieve a better-targeted effective therapy.

Extracellular matrix metalloproteinase inducer (EMMPRIN), also known as CD147 or basigin, is a multifunctional cell surface glycoprotein that belongs to the immunoglobulin superfamily. It is highly expressed on the surface of malignant cells and acts as an important mediator of tumor cell invasion via stimulation of matrix metalloproteinases (MMPs) production, tumor cell-induced angiogenesis via the stimulation of vascular endothelial growth factor production and multidrug resistance via hyaluronan-induced signaling (6). Recent studies have reported its association with poor prognostic features and aggressive phenotype in several types of tumors, 
including prostate cancer (7), tongue (8), uterine cervix (9) and esophageal (10) SCCs.

Fascin is a $55-\mathrm{kDa}$ actin-bundling protein that plays a pivotal role in cell motility, migration and adhesion. There are three highly related forms of fascin proteins: Fascin 1 (also known as fascin), expressed by mesenchymal and nervous tissues. The other two forms, fascin-2 and -3 , are expressed in the retinal photoreceptor cells and testes, respectively (11). Fascin is overexpressed in a wide variety of tumors including esophageal (12), prostatic (13) and ovarian (14) carcinomas and usually correlates with aggressive features and poor prognosis. However, it is usually absent or extremely underexpressed in normal epithelia (11).

A number of studies are available on EMMPRIN (15-18) and fascin (19-21) expression in the carcinoma of the urinary bladder. These studies have reported high EMMPRIN and fascin expression scores in invasive bladder urothelial carcinoma and suggested that EMMPRIN and fascin overexpression may be an indicator of aggressive TCC. None of these studies, however, has assessed the expression of the two proteins in SCC of the urinary bladder or schistosomiasis-associated bladder carcinomas.

EMMPRIN has been reported as an inducer of MMPs and causes tumor invasion and enhances the tumor metastatic potential (6). Xie et al (22) reported that the effect of fascin on cell invasion also depended on the activation of MMP-2 and -9. Although the detailed pathway has yet to be fully established, we hypothesized the presence of a possible correlation between EMMPRIN and fascin expression that may have a synergistic effect on bladder cancer.

In the present study, an immunohistochemical evaluation of EMMPRIN and fascin proteins in TCC and SCC of the urinary bladder was conducted in a cohort of Egyptian patients. Expression of EMMPRIN and fascin was also correlated with the schistosomal status, as well as with other available clinicopathological characteristics. Furthermore, we investigated the presence of possible correlations between EMMPRIN and fascin expression in this series of urinary bladder carcinomas.

\section{Materials and methods}

Patients and tissue specimens. This prospective study included 125 patients (106 males and 19 females) referred to the El-Minia University Hospital (Minia, Egypt) for the management of bladder tumor between January, 2009 and July, 2011. The patients were subjected to full clinical evaluations, laboratory investigations and imaging studies. Patient age ranged from 45 to 70 years (mean, $56.97 \pm 6.38$ years; median, 56 years). Tumor specimens were obtained by radical cystectomy or transurethral resection biopsy and were sent to the pathology department for histopathological examination. Only biopsies containing muscle tissue were included, in order that muscle invasion by the tumor could be evaluated.

Histopathology. Hematoxylin and eosin (H\&E)-stained sections were prepared and examined according to standard histopathological examination (23) to confirm tumor type, grade and pathological stage. Pathological examination showed TCC in 86 and SCC in 39 cases. Bilharzial infestation
Table I. Patient clinicopathological characteristics.

\begin{tabular}{lcc}
\hline $\begin{array}{l}\text { Clinicopathological } \\
\text { characteristics }\end{array}$ & $\begin{array}{c}\text { TCC } \\
\mathrm{n}=86 / 125(68.8 \%)\end{array}$ & $\begin{array}{c}\text { SCC } \\
\mathrm{n}=39 / 125(31.2 \%)\end{array}$ \\
\hline $\begin{array}{l}\text { Age (years, } \%) \\
\leq 50\end{array}$ & $9(10.5 \%)$ & $15(38.5 \%)$ \\
$>50$ & $77(89.5 \%)$ & $24(61.5 \%)$ \\
Gender & & \\
Male & $72(83.7 \%)$ & $34(87.2 \%)$ \\
Female & $14(16.3 \%)$ & $5(12.8 \%)$ \\
Grade & & \\
Low & $50(58.1 \%)$ & I $14(35.9 \%)$ \\
High & $36(41.9 \%)$ & II $17(43.6 \%)$ \\
& & III $8(20.5 \%)$ \\
Schistosomal status & & \\
Negative & $44(51.2 \%)$ & $10(25.6 \%)$ \\
Positive & $42(48.8 \%)$ & $29(74.4 \%)$ \\
Pathological stage & & \\
T1 & $18(20.9 \%)$ & 0 \\
T2 & $32(37.2 \%)$ & $6(15.4 \%)$ \\
T3 & $25(29.1 \%)$ & $21(53.8 \%)$ \\
T4 & $11(12.8 \%)$ & $12(30.8 \%)$ \\
\hline
\end{tabular}

TCC, transitional cell carcinoma; SCC, squamous cell carcinoma.

was evaluated by detection of bilharzial ova in tumor tissue or adjacent non-neoplastic tissue or from the data already provided in the patients' records.

Immunohistochemistry (IHC). Streptavidin-biotin immunoperoxidase complex procedure was applied for immunostaining. In brief, $4 \mu \mathrm{m}$ sections were deparaffinized with xylene, and rehydrated through graded alcohol. Endogenous peroxidase activity was blocked by incubation with $0.3 \%$ hydrogen peroxide in methanol for $30 \mathrm{~min}$. Antigen retrieval was achieved by microwave treatment in sodium citrate buffer $(0.01 \mathrm{M}$, pH 6.0) for $10 \mathrm{~min}$. Tissue sections were then incubated with monoclonal antibodies for EMMPRIN (mouse monoclonal sc-21746 antibody; dilution 1:100; Santa Cruz Biotechnology, Inc., Santa Cruz, CA, USA) and fascin (mouse monoclonal sc-21743 antibody; dilution 1:100; Santa Cruz Biotechnology, Inc.) for $1 \mathrm{~h}$, followed by biotinylated secondary antibody for $30 \mathrm{~min}$ at room temperature. Visualization of the reaction was performed with an avidin-biotin complex immunoperoxidase system using 3,3' diaminobenzidine (DAB) as a chromogen. Sections were then counterstained with hematoxylin, dehydrated, cleared and mounted with distyrene, plasticizer and xylene (DPX).

Positive and negative control. Each staining batch included positive and negative control sections. Negative control sections were treated with phosphate-buffered saline (PBS) instead of primary antibody. Sections of positive controls used were invasive ductal breast carcinoma for EMMPRIN and high-grade breast carcinomas for fascin. 

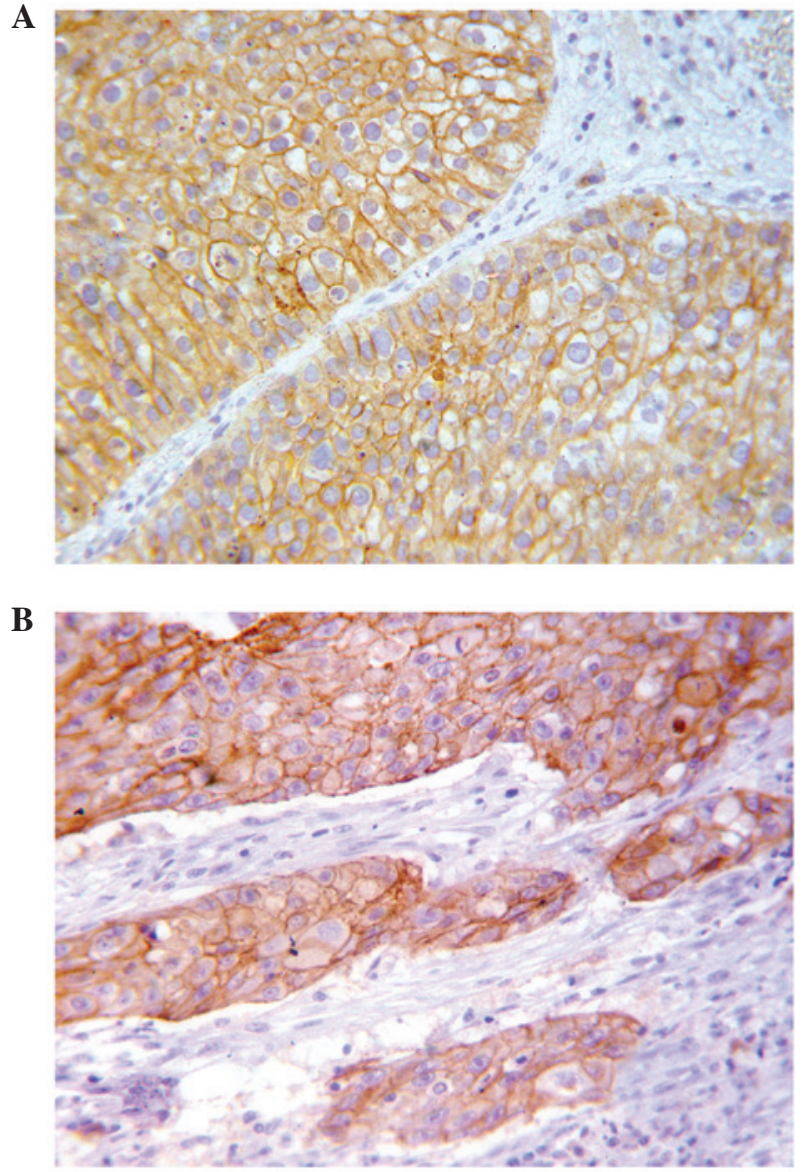

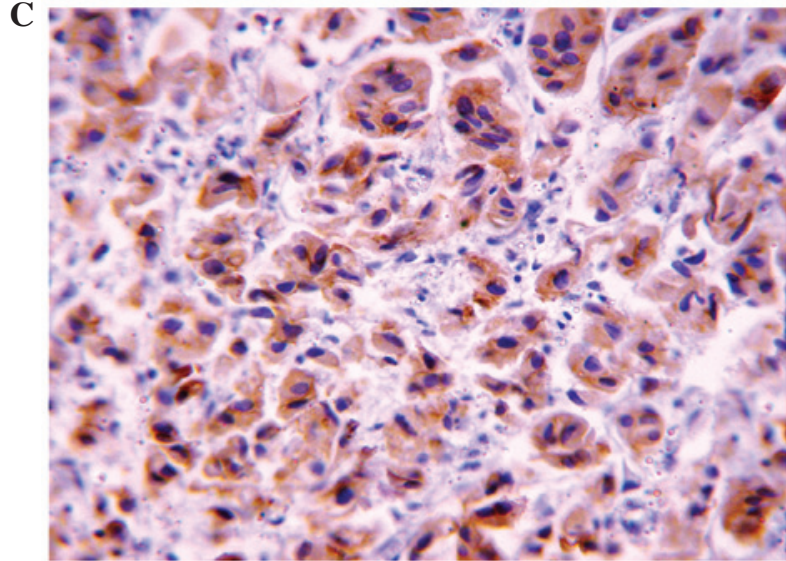

D

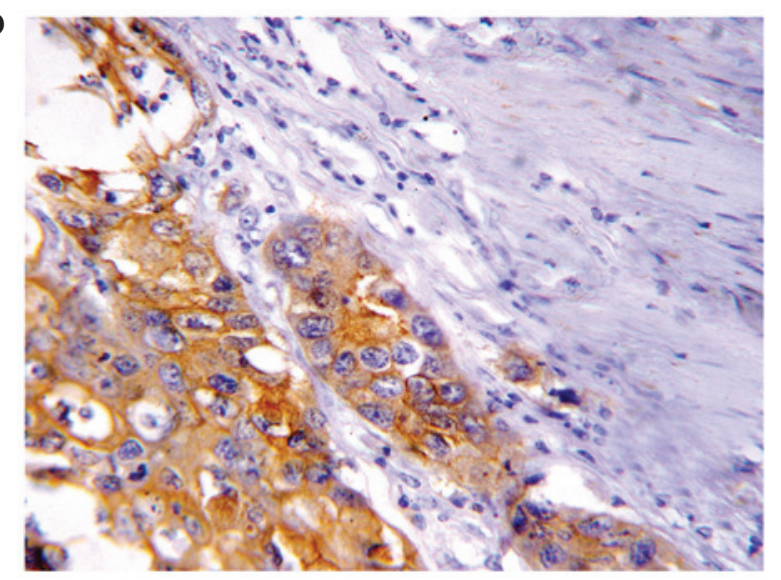

Figure 1. Immunohistochemical image of expression of EMMPRIN and fascin in invasive urinary bladder carcinoma. High membranous expression scores of EMMPRIN in (A) TCC and (B) SCC cases. High fascin cytoplasmic expression scores in (C) TCC and (D) SCC cases. Images were analyzed using immunohistochemistry, 3,3' diaminobenzidine chromogen and hematoxylin counterstaining; magnification, $x$ 400). EMMPRIN, extracellular matrix metalloproteinase inducer; TCC, transitional cell carcinoma; SCC, squamous cell carcinoma.

Scoring system. The levels of EMMPRIN and fascin protein expression were evaluated using a semi-quantitative scoring system, which was performed as described in previous studies $(19,24)$. Each slide was evaluated for the intensity of the staining and the percentage of tumor cells stained positive. Immunoreactivity was assessed by two pathologists. The correlation of their findings was high and in case of discrepancies, a consensus was reached by joint evaluation. The extent of the staining was scored as: $<25 \%$ of tumor cells stained, 1 ; $25-50 \%$ of the tumor cells stained positive, $2 ; 51-75 \%$ of the tumor cells stained positive, 3 and $>75 \%$ of the tumor cells stained positive, 4 . Staining intensity was graded on a scale of 0-3: no staining, 0 ; weak, 1 ; moderate, 2 ; or intense staining, 3 . The combined score was calculated by multiplying the intensity and percentage scores yielding an overall score range of 0 -12. A final score of $\leq 2$ was considered negative.

Statistical analysis. Statistical analysis was conducted using the SPSS software version 11.0. Raw data were compiled and used to determine the means \pm standard deviations (SDs), median and ranges of various variables. Non-parametric statistics were performed to evaluate the association between EMMPRIN and fascin and various clinicopathological characteristics. The Kruskal-Wallis test was carried out to assess the differences of expression in the clinicopathological variables with $>2$ groups, and the Mann-Whitney test was used to evaluate difference of expression in the dichotomous variables. The Chi-square and Fisher's exact tests were used to compare categorical variables. $\mathrm{P} \leq 0.05$ was considered to indicate a statistically significant difference.

\section{Results}

Clinicopathological data. Table I shows a summary of the clinicopathological characteristics for the cases included in this study. A total of 125 patients with primary bladder cancer were included, $68.8 \%$ of whom were TCC, while $31.2 \%$ of were SCC. A significantly lower patient age in SCC cases compared to TCC cases was observed $(\mathrm{P}<0.001)$. The mean patient age was $52.51 \pm 4.26$ and 59.00 \pm 6.17 years in SCC and TCC, respectively, with male predominance in the two types. Evidence of schistosomiasis was noted in $71 / 125(56.8 \%)$ of patients. A significantly $(\mathrm{P}=0.008)$ lower frequency of associated schistosomiasis was noted in TCC compared to SCC cases. Regarding invasiveness, all the SCC and $79.1 \%$ of TCC cases were muscle invasive (T2, T3 and T4) at the time of diagnosis.

Expression of EMMPRIN and fascin in normal and neoplastic bladder tissues. EMMPRIN and fascin immunoreactivity was undetectable in normal urothelium. However, EMMPRIN and fascin expression was detectable on the cell membrane (Fig. 1A and B) and cytoplasm (Fig. 1C and D) 
Table II. Correlation of EMMPRIN and fascin expression and clinicopathological characteristics of the 86 examined TCC cases.

\begin{tabular}{|c|c|c|c|c|}
\hline Clinicopathological characteristics & $\begin{array}{c}\text { EMMPRIN } \\
\text { Mean } \pm \text { SD } \\
\text { Median (min-max) }\end{array}$ & P-value & $\begin{array}{c}\text { Fascin } \\
\text { Mean } \pm \text { SD } \\
\text { Median (min-max) }\end{array}$ & P-value \\
\hline \multicolumn{5}{|l|}{ Age (years) } \\
\hline$\leq 50$ & $\begin{array}{c}5.22 \pm 4.46 \\
6(0-12)\end{array}$ & 0.690 & $\begin{array}{c}7.66 \pm 3.70 \\
9(0-12)\end{array}$ & 0.453 \\
\hline$>50$ & $\begin{array}{c}5.24 \pm 4.15 \\
6(0-12)\end{array}$ & & $\begin{array}{c}6.67 \pm 3.98 \\
8(0-12)\end{array}$ & \\
\hline \multicolumn{5}{|l|}{ Gender } \\
\hline Male & $\begin{array}{c}5.75 \pm 4.13 \\
6(0-12)\end{array}$ & 0.01 & $\begin{array}{c}7.05 \pm 3.90 \\
8(0-12)\end{array}$ & 0.127 \\
\hline Female & $\begin{array}{c}2.64 \pm 3.31 \\
0(0-9)\end{array}$ & & $\begin{array}{c}5.35 \pm 4.03 \\
5(0-12)\end{array}$ & \\
\hline \multicolumn{5}{|l|}{ Schistosomiasis association } \\
\hline Negative & $\begin{array}{c}5.13 \pm 4.00 \\
6(0-12)\end{array}$ & 0.715 & $\begin{array}{c}6.50 \pm 3.86 \\
8(0-12)\end{array}$ & 0.549 \\
\hline Positive & $\begin{array}{c}5.35 \pm 4.36 \\
6(0-12)\end{array}$ & & $\begin{array}{c}7.07 \pm 4.06 \\
8(0-12)\end{array}$ & \\
\hline \multicolumn{5}{|l|}{ Grade } \\
\hline Low & $\begin{array}{c}4.04 \pm 3.90 \\
4(0-12)\end{array}$ & 0.003 & $\begin{array}{c}6.32 \pm 3.91 \\
6(0-12)\end{array}$ & 0.190 \\
\hline High & $\begin{array}{c}6.91 \pm 3.96 \\
8(0-12)\end{array}$ & & $\begin{array}{c}7.41 \pm 3.95 \\
8(0-12)\end{array}$ & \\
\hline \multicolumn{5}{|l|}{ Pathological stage } \\
\hline $\mathrm{T} 1$ & $\begin{array}{c}1.38 \pm 2.45 \\
0(0-9)\end{array}$ & $<0.001$ & $\begin{array}{c}3.22 \pm 3.43 \\
2(0-12)\end{array}$ & $<0.001$ \\
\hline $\mathrm{T} 2$ & $\begin{array}{c}4.78 \pm 3.76 \\
5(0-12)\end{array}$ & & $\begin{array}{c}6.15 \pm 3.52 \\
6(0-12)\end{array}$ & \\
\hline $\mathrm{T} 3$ & $\begin{array}{c}7.08 \pm 3.51 \\
8(0-12)\end{array}$ & & $\begin{array}{c}8.64 \pm 2.79 \\
9(0-12)\end{array}$ & \\
\hline $\mathrm{T} 4$ & $\begin{array}{c}8.72 \pm 3.77 \\
9(0-12)\end{array}$ & & $\begin{array}{c}10.18 \pm 3.12 \\
12(2-12)\end{array}$ & \\
\hline
\end{tabular}

Kruskal-Wallis and Mann-Whitney tests served as the test of significance. $\mathrm{P} \leq 0.05$, statistically significant. TCC, transitional cell carcinoma; EMMPRIN, expression of extracellular matrix metalloproteinase inducer; SD, standard deviation.

in 71.2 and $83.2 \%$ of bladder carcinomas, respectively. A significantly higher EMMPRIN mean of expression was detected in SCC [mean \pm SD $6.97 \pm 4.21$, median $8(0-12)]$ compared to TCC [mean \pm SD 5.24 \pm 4.16 , median $6(0-12)]$ cases $(\mathrm{P}=0.031)$. Similarly, a higher fascin mean of expression was noted in SCC [mean \pm SD 8.35 \pm 3.03 , median 8 (2-12)] compared to TCC [mean \pm SD $6.77 \pm 3.95$, median 8 (0-12)] cases, however, such a difference was not statistically significant $(\mathrm{P}=0.053)$.

Expression of EMMPRIN and fascin in TCC. Table II summarizes the immunohistochemical results of ECMMPRIN and fascin expression in correlation with various clinicopathological parameters in TCC cases.

Regarding EMMPRIN expression, significant positive associations between EMMPRIN overexpression and gender, tumor grade and pathological stage were identified $(\mathrm{P}=0.01$, 0.003 and $<0.001$, respectively). EMMPRIN expression was found to be significantly associated with an increasing invasiveness of the tumor. Non-muscle invasive urothelial carcinomas ( $\mathrm{pT} 1)$ were strongly correlated with negative or low EMMPRIN expression scores compared to muscle invasive tumors $(\mathrm{pT} 2-4)(\mathrm{P}<0.001)$. Similarly, low-grade tumors were associated with negative or low EMMPRIN expression scores, whereas high-grade tumors showed higher expression scores. No significant associations were observed regarding patient age and schistosomal status. Concerning fascin expression, statistical analysis demonstrated a significant positive association between high fascin immunostaining scores and tumor pathological stage $(\mathrm{P}<0.001)$, i.e., the more the increase of the tumor stage, the higher the fascin expression score. A highly significant difference was identified between 
Table III. Correlation of EMMPRIN and fascin expression and clinicopathological characteristics of the 39 examined SCC cases.

\begin{tabular}{|c|c|c|c|c|}
\hline Clinicopathological characteristics & $\begin{array}{c}\text { EMMPRIN } \\
\text { Mean } \pm \text { SD } \\
\text { Median (min-max) }\end{array}$ & P-value & $\begin{array}{c}\text { Fascin } \\
\text { Mean } \pm \text { SD } \\
\text { Median (min-max) }\end{array}$ & P-value \\
\hline \multicolumn{5}{|l|}{ Age (years) } \\
\hline$\leq 50$ & $\begin{array}{c}5.66 \pm 4.02 \\
6(0-12)\end{array}$ & 0.151 & $\begin{array}{c}7.40 \pm 3.83 \\
8(2-12)\end{array}$ & 0.300 \\
\hline$>50$ & $\begin{array}{c}7.79 \pm 4.20 \\
8(0-12)\end{array}$ & & $\begin{array}{l}8.95 \pm 2.31 \\
8.5(4-12)\end{array}$ & \\
\hline \multicolumn{5}{|l|}{ Gender } \\
\hline Male & $\begin{array}{c}7.08 \pm 4.21 \\
8(0-12)\end{array}$ & 0.670 & $\begin{array}{c}8.06 \pm 3.12 \\
8(2-12)\end{array}$ & 0.477 \\
\hline Female & $\begin{array}{c}6.20 \pm 4.60 \\
6(0-12)\end{array}$ & & $\begin{array}{c}9.71 \pm 2.36 \\
9(6-12)\end{array}$ & \\
\hline \multicolumn{5}{|l|}{ Schistosomiasis association } \\
\hline Negative & $\begin{array}{c}5.50 \pm 4.00 \\
5(0-12)\end{array}$ & 0.186 & $\begin{array}{c}7.30 \pm 3.33 \\
8(2-12)\end{array}$ & 0.182 \\
\hline Positive & $\begin{array}{c}7.48 \pm 4.23 \\
8(0-12)\end{array}$ & & $\begin{array}{c}8.72 \pm 2.90 \\
9(2-12)\end{array}$ & \\
\hline \multicolumn{5}{|l|}{ Grade } \\
\hline I & $\begin{array}{c}4.64 \pm 4.25 \\
5(0-12)\end{array}$ & 0.002 & $\begin{array}{c}7.28 \pm 3.04 \\
8(2-12)\end{array}$ & 0.339 \\
\hline II & $\begin{array}{c}7.05 \pm 3.59 \\
8(0-12)\end{array}$ & & $\begin{array}{c}9.00 \pm 2.80 \\
9(2-12)\end{array}$ & \\
\hline III & $\begin{array}{c}10.87 \pm 2.23 \\
12(6-12)\end{array}$ & & $\begin{array}{l}8.87 \pm 3.35 \\
8.5(2-12)\end{array}$ & \\
\hline \multicolumn{5}{|l|}{ Pathological stage } \\
\hline $\mathrm{T} 1$ & $\begin{array}{l}- \\
-\end{array}$ & 0.004 & $\begin{array}{l}- \\
-\end{array}$ & 0.001 \\
\hline $\mathrm{T} 2$ & $\begin{array}{c}3.50 \pm 4.18 \\
2(0-9)\end{array}$ & & $\begin{array}{c}4.33 \pm 1.96 \\
5(2-6)\end{array}$ & \\
\hline T3 & $\begin{array}{c}6.28 \pm 3.63 \\
6(0-12)\end{array}$ & & $\begin{array}{c}8.42 \pm 2.76 \\
8(2-12)\end{array}$ & \\
\hline $\mathrm{T} 4$ & $\begin{array}{c}9.91 \pm 3.52 \\
12(2-12)\end{array}$ & & $\begin{array}{l}10.25 \pm 1.86 \\
10.5(8-12)\end{array}$ & \\
\hline
\end{tabular}

Kruskal-Wallis and Mann-Whitney tests served as the test of significance. $\mathrm{P} \leq 0.05$, statistically significant. SCC; squamous cell carcinoma EMMPRIN, expression of extracellular matrix metalloproteinase inducer; SD, standard deviation.

non-muscle invasive (pT1) and muscle invasive (pT2-4) TCC cases $(\mathrm{P}<0.001)$. No significant associations were detected between fascin expression and other clinicopathological characteristics.

EMMPRIN and fascin expression in SCC cases. Table III shows the immunohistochemical results of EMMPRIN and fascin expression in correlation with various clinicopathological characteristics in SCC cases.

Regarding EMMPRIN expression, significant positive associations between EMMPRIN overexpression and tumor grade and pathological stage were identified $(\mathrm{P}=0.002$ and 0.004 , respectively). No significant associations were observed regarding patient age, gender or schistosomal status. Concerning fascin expression, statistical analysis demonstrated a significant positive association between high fascin immunostaining scores and tumor pathological stage $(\mathrm{P}=0.001)$, whereas no significant associations were detected with other clinicopathological characteristics.

Correlation between EMMPRIN and fascin in urinary bladder carcinomas. The correlation between EMMPRIN and fascin immunostaining scores is shown in Fig. 2. Overall, a significant positive correlation was observed between fascin and EMMPRIN expression in urinary bladder carcinomas (Spearman's rho correlation coefficient $(\mathrm{r})=0.500, \mathrm{P}<0.001)$. A positive correlation was also detected in TCC and SCC cases ( $r=0.535, \mathrm{P}<0.001$ and $\mathrm{r}=0.372, \mathrm{P}=0.020$, respectively) 
Table IV. EMMPRIN and fascin immunophenotypes in correlation with stage in urinary bladder carcinomas $(\mathrm{P}=<0.001)$.

Pathological stage

\begin{tabular}{lccccc} 
Immunophenotype & Frequency $(\%)$ & $\mathrm{T} 1 \mathrm{n}=18(\%)$ & $\mathrm{T} 2 \mathrm{n}=38(\%)$ & $\mathrm{T} 3 \mathrm{n}=46(\%)$ & $\mathrm{T} 4 \mathrm{n}=23(\%)$ \\
\hline $\begin{array}{l}\text { Both negative } \\
\begin{array}{l}\text { EMMPRIN-positive/ } \\
\text { fascin-negative }\end{array}\end{array}$ & $14(11.2)$ & $10(55.6)$ & $3(7.9)$ & - & $1(4.3)$ \\
$\begin{array}{l}\text { EMMPRIN-negative/ } \\
\text { fascin-positive }\end{array}$ & $7(5.6)$ & $1(5.6)$ & $4(10.5)$ & $2(4.3)$ & - \\
\begin{tabular}{l} 
Both positive \\
\hline
\end{tabular} & $22(17.6)$ & $4(22.2)$ & $10(26.3)$ & $7(15.2)$ & $1(4.3)$ \\
\hline
\end{tabular}

Chi-square and Fischer's exact tests served as the test of significance. $\mathrm{P} \leq 0.05$, statistically significant. EMMPRIN, expression of extracellular matrix metalloproteinase inducer.

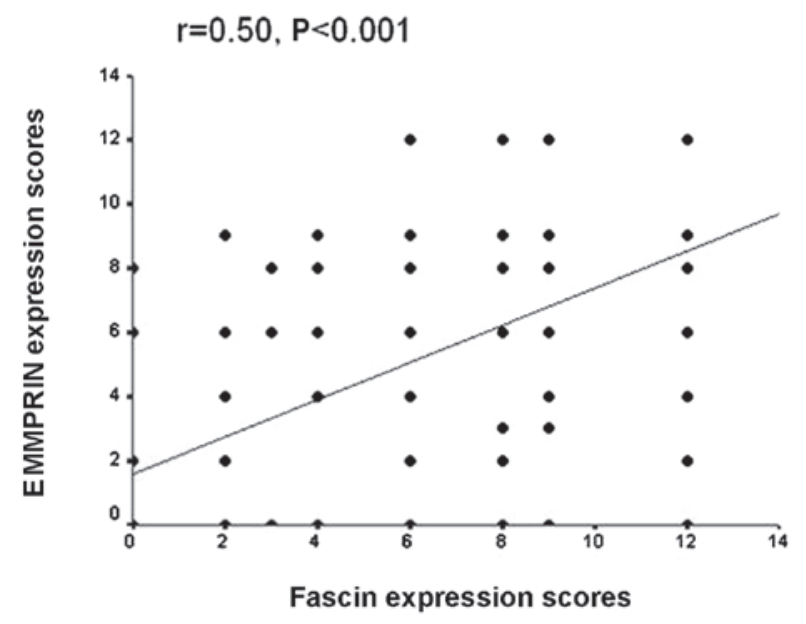

Figure 2. Significant positive correlation between EMMPRIN and fascin expression in urinary bladder carcinoma. EMMPRIN, expression of extracellular matrix metalloproteinase inducer.

Combined EMMPRIN and fascin immunophenotype and their correlation with pathological stage. The frequency of varying combined EMMPRIN and fascin immunoprofiles and their correlation with pathological stage is shown in Table IV. A highly significant association was found between various tumor immunoprofiles and pathological stage $(\mathrm{P}=<0.001)$. The EMMPRIN-/fascin-positive immunophenotype was closely associated with $\mathrm{T} 3$ and $\mathrm{T} 4$ tumors, while a great proportion of T1 tumors was negative for the two markers.

\section{Discussion}

During the past decade, certain changes have been reported in the features of bladder cancer in Egypt. Higher incidence rates of TCC compared to SCC cases, notable increases in the patient mean age and a marked decrease in the incidence of associated schistosomiasis were reported $(2,3)$. In agreement with those studies, the present study showed a markedly higher frequency rate of TCC (68.8\%) compared to SCC (31.2\%) cases and a relatively higher patient mean age. Evidence of schistosomiasis was found only in $56.8 \%$ of the patients. The present study also showed a significantly lower frequency of associated schistosomiasis in TCC compared to SCC cases, as well as a significantly lower patient age in SCC compared to TCC cases, with a male to female predominance in the two types of carcinoma, consistent with the findings of a previous report (2). Notably, the SCC specimens included in this study were classified as muscle-invasive tumors, indicating the more aggressive nature of SCC.

Various molecular pathways that have been found to be associated with bladder cancer are currently available. Recent studies have already identified the pivotal role of EMMPRIN (15-18) and fascin (19-21) proteins in urothelial carcinoma.

Regarding EMMPRIN expression, the present study showed a positive EMMPRIN expression in $71.2 \%$ of bladder carcinomas, while it was undetectable in normal urothelium. This is in agreement with previous findings (15-18).

The association between EMMPRIN overexpression and tumor progression has been documented. EMMPRIN overexpression induces tumor cell invasion and metastasis via MMPs production (6), while its silencing suppressed the proliferation ability of prostate carcinoma cell line LNCAP and bladder carcinoma cell line J82, suggesting its involvement in tumor cell proliferatin and growth (16). The present study has demonstrated significant positive associations between high EMMPRIN expression scores and advanced $\mathrm{pT}$ stage and a high grade in TCC and SCC cases. In TCC, EMMPRIN was found to be differentially expressed in muscle-invasive tumors (pT2-4) compared to non-muscle invasive (pT1) tumors with a progressive increase in its expression from pT1 to pT4. Moreover, high-grade carcinomas, compared to low-grade tumors, showed significantly higher expression scores. In SCC cases, the tumors were muscle invasive, however, EMMPRIN expression was closely associated with tumor stage, with an obvious increase from pT2 to pT4. Similarly, a strong association between its expression and SCC grade was identified, where grade III carcinomas were associated with a significantly increased expression compared to well-differentiated tumors. These findings are in concordance with those of previous studies in urothelial carcinomas $(15,16,18)$ and SCC in other tissues $(8,10)$.

By contrast, in their study Afonso et al (17) found no significant association of EMMPRIN with regard to clini- 
copathological parameters or patient outcome, which may be attributable to different case population, different scoring system and cut-off points used by various studies. However, Afonso et al (17) found that EMMPRIN expression added a predictive power of outcome to pathological stage: patients with pT3/pT4 tumors had a median overall survival time of 14.7 months, which was significantly reduced to 9.2 months if the tumors were EMMPRIN-positive.

Regarding fascin expression, $83.2 \%$ of the bladder carcinomas exhibited positive immunoreactivity, while no notable expression was observed in normal urothelium adjacent to carcinoma. Similarly, fascin expression was either low or absent in normal epithelia, while it was upregulated at mRNA and protein levels in several types of cancers including urothelial carcinoma of the bladder $(19,20)$.

Invasive and metastatic potential of tumor cells is often associated with actin cytoskeleton rearrangements involving several types of actin cross-linking proteins, of which fascin is the key component. Fascin overexpression is also suggested to disrupt epithelial junctions and thus to increase the invasive and metastatic potential of malignant cells (25). In the present study, overexpression of the fascin protein was significantly correlated with advanced tumor stage in the TCC and SCC cases. A highly significant difference was found in fascin expression between non-muscle invasive tumors and muscle-invasive TCC cases. Additionally, muscle-invasive tumors (pT2-4) showed a stepwise increase in fascin expression scores in the two tumor types. Previous studies also reported a significant association between high fascin expression and advanced tumor pathological stage with a progressive increase of its expression from superficial to deeply invasive urothelial carcinomas of the urinary bladder $(19,26)$.

No significant associations were observed with regard to tumor grade or other clinicopathological parameters. This lack of significant association between fascin expression and tumor grade noted in the present study confirmed the findings of previous studies $(19,20,26)$.

The present study shows no significant association between the presence of schistosomiasis and overexpression of EMMPRIN and fascin. No significant differences regarding EMMPRIN and fascin expression between schistosomiasisassociated and non-schistosomiasis-associated tumors was observed, suggesting that schistosomiasis may not be important in the upregulation of the two proteins in bladder cancer.

Various subtypes of bladder carcinomas represent different molecular alterations and biological behaviors (27). Notably, significantly higher EMMPRIN expression scores were found in SCC compared to TCC cases. Additionally, there was a tendency for higher fascin expression scores in SCC compared to TCC cases, a finding that may be attributable to the more aggressive and muscle-invasive nature of SCC of the bladder $(27,28)$. The differential expression of these markers in SCC and TCC also suggest an association between tumor behavior and their immunoreactivity.

In the literature, a positive correlation between EMMPRIN and fascin in renal (29) and colorectal (30) carcinomas was reported. However, the correlation between EMMPRIN and fascin proteins in bladder carcinoma has not been previously investigated. The present study showed a significant positive correlation between EMMPRIN and fascin expression in urinary bladder carcinomas. This finding, together with the findings of previous studies suggest a correlation between the two proteins that may induce a synergistic effect during tumor progression and invasion.

The main roles of EMMPRIN and fascin in tumor progression depend on MMPs and fascin-actin interactions, which cause tumor cell protrusion, invasion and migration $(6,11,22)$. Therefore, EMMPRIN and fascin were expected to be significantly correlated with $\mathrm{T}$ stages. In this context, we have characterized the joint expression of EMMPRIN with fascin to identify various immunoprofiles of EMMPRIN with fascin expression and their implication on pathological stage. Notably, the majority of T3 and T4 tumors showed combined positive expression for the two markers. By contrast, a substantial proportion of T1 tumors were negative for the two markers. Our finding suggests that the co-localization of EMMPRIN with fascin in bladder cancer emphasizes the possible cooperative involvement of these markers in cancer invasion.

In conclusion, the present study demonstrated that EMMPRIN and fascin are highly expressed in bladder carcinomas, whereas neither EMMPRIN nor fascin was detectable in the normal adjacent epithelia. High expression scores were correlated with bladder cancer progression and aggressive phenotype. The present study also found no statistically significant differences regarding EMMPRIN and fascin expression in schistosomiasis-associated and non-associated bladder carcinomas. A statistically significant positive correlation between EMMPRIN and fascin expression was observed in this series. Combined positive expression of the two markers was strongly associated with advanced stage, suggesting a correlation between the two proteins that may induce a synergistic effect during tumor progression and invasion. The correlation between EMMPRIN with fascin should be clarified on a wider scale of tumors. Overexpression of EMMPRIN with fascin are potentially significant biomarkers of urinary bladder cancer aggressiveness that may help surgeons to identify patients who are likely to benefit from a personalized therapeutic regimen and more aggressive treatment strategies.

\section{References}

1. Ploeg M, Aben KK and Kiemeney LA: The present and future burden of urinary bladder cancer in the world. World J Urol 27: 289-293, 2009.

2. Salem HK and Mahfouz S: Changing patterns (age, incidence, and pathologic types) of schistosoma-associated bladder cancer in Egypt in the past decade. Urology 79: 379-383, 2012.

3. Mokhtar N, Gouda I and Adel I (eds): Cancer pathology registry 2003-2004 and time trend analysis. NCI, El Sheraa Press, Cairo, 2007.

4. Cheng L, Montironi R, Davidson DD and Lopez-Beltran A: Staging and reporting of urothelial carcinoma of the urinary bladder. Mod Pathol 22 (Suppl 2): 70-95, 2009.

5. Als AB, Dyrskjøt L, von der Maase H, et al: Emmprin and survivin predict response and survival following cisplatin-containing chemotherapy in patients with advanced bladder cancer. Clin Cancer Res 13: 4407-4414, 2007.

6. Yan L, Zucker S and Toole BP: Roles of the multifunctional glycoprotein, emmprin (basigin; CD147), in tumour progression. Thromb Haemost 93: 199-204, 2005.

7. Han ZD, Bi XC, Qin WJ, et al: CD147 expression indicates unfavourable prognosis in prostate cancer. Pathol Oncol Res 15: 369-374, 2009

8. Huang Z, Huang H, Li H, et al: EMMPRIN expression in tongue squamous cell carcinoma. J Oral Pathol Med 38: 518-523, 2009. 
9. Yu W, Liu J, Xiong X, et al: Expression of MMP9 and CD147 in invasive squamous cell carcinoma of the uterine cervix and their implication. Pathol Res Pract 205: 709-715, 2009.

10. Zhu S, Li Y, Mi L, et al: Clinical impact of HAb18G/CD147 expression in esophageal squamous cell carcinoma. Dig Dis Sci 56: 3569-3576, 2011

11. Hashimoto Y, Skacel M and Adams JC: Roles of fascin in human carcinoma motility and signaling: prospects for a novel biomarker? Int J Biochem Cell Biol 37: 1787-1804, 2005.

12. Zhang H, Xu L, Xiao D, et al: Fascin is a potential biomarker for early-stage oesophageal squamous cell carcinoma. J Clin Pathol 59: 958-964, 2006.

13. Darnel AD, Behmoaram E, Vollmer RT, et al: Fascin regulates prostate cancer cell invasion and is associated with metastasis and biochemical failure in prostate cancer. Clin Cancer Res 15 : 1376-1383, 2009.

14. Lin CK, Chao TK, Yu CP, et al: The expression of six biomarkers in the four most common ovarian cancers: correlation with clinicopathological parameters. APMIS 117: 162-175, 2009.

15. Zhong WD, Chen QB, Ye YK, et al: Extracellular matrix metalloproteinase inducer expression has an impact on survival in human bladder cancer. Cancer Epidemiol 34: 478-482, 2010.

16. Han ZD, He HC, Bi XC, et al: Expression and clinical significance of CD147 in genitourinary carcinomas. J Surg Res 160: 260-267, 2010

17. Afonso J, Longatto-Filho A, Baltazar F, et al: CD147 overexpression allows an accurate discrimination of bladder cancer patients' prognosis. Eur J Surg Oncol 37: 811-817, 2011.

18. Wittschieber D, Stenzinger A, Klauschen F, et al: Decreased RECK and increased EMMPRIN expression in urothelial carcinoma of the bladder are associated with tumor aggressiveness. Pathobiology 78: 123-131, 2011.

19. Karasavvidou F, Barbanis S, Pappa D, et al: Fascin determination in urothelial carcinomas of the urinary bladder: a marker of invasiveness. Arch Pathol Lab Med 132: 1912-1915, 2008.
20. Bi J, Chen X, Zhang Y, et al: Fascin is a predictor for invasiveness and recurrence of urothelial carcinoma of bladder. Urol Oncol 30: 688-694, 2010

21. McKnight R, Cohen C and Siddiqui MT: Fascin stain as a potential marker of invasiveness in carcinomas of the urinary bladder: a retrospective study with biopsy and cytology correlation. Diagn Cytopathol 39: 635-640, 2011.

22. Xie JJ, Xu LY, Zhang HH, et al: Role of fascin in the proliferation and invasiveness of esophageal carcinoma cells. Biochem Biophys Res Commun 337: 355-362, 2005.

23. Eble JN, Sauter G, Epstein JI, Sesterhenn IA (eds): World Health Organization classification of tumors. Pathology and genetics of tumors of the urinary system and male genital organs. IARC Press, Lyon, pp90-109, 2004

24. Takikita M, Hu N, Shou JZ, et al: Fascin and CK4 as biomarkers for esophageal squamous cell carcinoma. Anticancer Res 31: 945-952, 2011.

25. Jayo A and Parsons M: Fascin: a key regulator of cytoskeletal dynamics. Int J Biochem Cell Biology 42: 1614-1617, 2010.

26. Tong GX, Yee H, Chiriboga L, et al: Fascin-1 expression in papillary and invasive urothelial carcinomas of the urinary bladder. Hum Pathol 36: 741-746, 2005.

27. Blaveri E, Simko JP, Korkola JE, et al: Bladder cancer outcome and subtype classification by gene expression. Clin Cancer Res 11: 4044-4055, 2005.

28. Shokeir AA: Squamous cell carcinoma of the bladder: pathology, diagnosis and treatment. BJU int 93: 216-220, 2004.

29. Tsai WC, Sheu LF, Nieh S, et al: Association of EMMPRIN and fascin expression in renal cell carcinoma: correlation with clinicopathological parameters. World J Urol 25: 73-80, 2007.

30. Jung EJ, Lee JH, Min BW, et al: Clinicopathologic significance of fascin, extracellular matrix metalloproteinase inducer, and ezrin expressions in colorectal adenocarcinoma. Indian J Pathol Microbiol 54: 32-36, 2011. 